\title{
Manifold Answer-Set Programs for Meta-Reasoning ${ }^{\star}$
}

\author{
Wolfgang Faber ${ }^{1}$ and Stefan Woltran ${ }^{2}$ \\ 1 University of Calabria, Italy \\ wf@wfaber.com \\ 2 Vienna University of Technology, Austria \\ woltran@dbai.tuwien.ac.at
}

\begin{abstract}
In answer-set programming (ASP), the main focus usually is on computing answer sets which correspond to solutions to the problem represented by a logic program. Simple reasoning over answer sets is sometimes supported by ASP systems (usually in the form of computing brave or cautious consequences), but slightly more involved reasoning problems require external postprocessing. Generally speaking, it is often desirable to use (a subset of) brave or cautious consequences of a program $P_{1}$ as input to another program $P_{2}$ in order to provide the desired solutions to the problem to be solved. In practice, the evaluation of the program $P_{1}$ currently has to be decoupled from the evaluation of $P_{2}$ using an intermediate step which collects the desired consequences of $P_{1}$ and provides them as input to $P_{2}$. In this work, we present a novel method for representing such a procedure within a single program, and thus within the realm of ASP itself. Our technique relies on rewriting $P_{1}$ into a so-called manifold program, which allows for accessing all desired consequences of $P_{1}$ within a single answer set. Then, this manifold program can be evaluated jointly with $P_{2}$ avoiding any intermediate computation step. For determining the consequences within the manifold program we use weak constraints, which is strongly motivated by complexity considerations. As an application, we present an encoding for computing the ideal extension of an abstract argumentation framework.
\end{abstract}

\section{Introduction}

In the last decade, Answer Set Programming (ASP) [1,2], also known as A-Prolog $[3,4]$, has emerged as a declarative programming paradigm. ASP is well suited for modelling and solving problems which involve common-sense reasoning, and has been fruitfully applied to a wide variety of applications including diagnosis, data integration, configuration, and many others. Moreover, the efficiency of the latest tools for processing ASP programs (so-called ASP solvers) reached a state that makes them applicable for problems of practical importance [5]. The basic idea of ASP is to compute answer sets (usually stable models) of a logic program from which the solutions of the problem encoded by the program can be obtained.

However, frequently one is interested not only in the solutions per se, but rather in reasoning tasks that have to take some or even all solutions into account. As an example, consider the problem of database repair, in which a given database instance does

\footnotetext{
* This work was supported by the Vienna Science and Technology Fund (WWTF), grant ICT08028, and by M.I.U.R. within the Italia-Austria internazionalization project "Sistemi basati sulla logica per la rappresentazione di conoscenza: estensioni e tecniche di ottimizzazione.".
} 
not satisfy some of the constraints imposed in the database. One can attempt to modify the data in order to obtain a consistent database by changing as little as possible. This will in general yield multiple possibilities and can be encoded conveniently using ASP (see, e.g., [6]). However, usually one is not interested in the repairs themselves, but in the data which is present in all repairs. For the ASP encoding, this means that one is interested in the elements which occur in all answer sets; these are also known as cautious consequences. Indeed, ASP systems provide special interfaces for computing cautious consequences by means of query answering. But sometimes one has to do more, such as answering a complex query over the cautious consequences (not to be confused with complex queries over answer sets). So far, ASP solvers provide no support for such tasks. Instead, computations like this have to be done outside ASP systems, which hampers usability and limits the potential of ASP.

In this work, we tackle this limitation by providing a technique, which transforms an ASP program $P$ into a manifold program $M_{P}$ which we use to identify all consequences of a certain type ${ }^{3}$ within a single answer set. The main advantage of the manifold approach is that the resulting program can be extended by additional rules representing a query over the brave (or cautious, definite) consequences of the original program $P$, thereby using ASP itself for this additional reasoning. In order to identify the consequences, we use weak constraints [8], which are supported by the ASP-solver DLV [9]. Weak constraints have been introduced to prefer a certain subset of answer sets via penalization. Their use for computing consequences is justified by a complexity-theoretic argument: One can show that computing consequences is complete for the complexity classes $\mathrm{FP}_{\|}^{\mathrm{NP}}$ or $\mathrm{FP}_{\|}^{\Sigma_{2}^{P}}$ (depending on the presence of disjunction), for which also computing answer sets for programs with weak constraints is complete ${ }^{4}$, which means that an equivalent compact ASP program without these extra constructs does not exist, unless the polynomial hierarchy collapses. In principle, other preferential constructs similar to weak constraints could be used as well for our purposes, as long as they meet these complexity requirements.

We discuss two particular applications of the manifold approach. First, we specify an encoding which decides the SAT-related unique minimal model problem, which is closely related to closed-world reasoning [10]. The second problem stems from the area of argumentation (cf. [11] for an overview) and concerns the computation of the ideal extension [12] of an argumentation framework. For both problems we make use of manifold programs of well-known encodings (computing all models of a CNF-formula for the former application, computing all admissible extensions of an argumentation framework for the latter) in order to compute consequences. Extensions by a few more rules then directly provide the desired solutions, requiring little effort in total.

\footnotetext{
${ }^{3}$ We consider here the well-known concepts of brave and cautious consequence, but also definite consequence [7].

${ }^{4}$ The first of these results is fairly easy to see, for the second, it was shown [8] that the related decision problem is complete for the class $\Theta_{2}^{P}$ or $\Theta_{3}^{P}$, from which the $\mathrm{FP}_{\|}^{\mathrm{NP}}$ and $\mathrm{FP}_{\|}^{\Sigma_{2}^{P}}$ results can be obtained. Also note that frequently cited NP, $\Sigma_{2}^{P}$, and co-NP, $\Pi_{2}^{P}$ completeness results hold for brave and cautious query answering, respectively, but not for computing brave and cautious consequences.
} 
Organization and Main Results. After introducing the necessary background in the next section, we

- introduce in Section 3 the concept of a manifold program for rewriting propositional programs in such a way that all brave (resp. cautious, definite) consequences of the original program are collected into a single answer set;

- lift the results to the non-ground case (Section 4); and

- present applications for our technique in Section 5. In particular, we provide an ASP encoding for computing the ideal extension of an argumentation framework.

The paper concludes with a brief discussion of related and further work.

\section{Preliminaries}

In this section, we review the basic syntax and semantics of ASP with weak constraints, following [9], to which we refer for a more detailed definition.

An atom is an expression $p\left(t_{1}, \ldots, t_{n}\right)$, where $p$ is a predicate of arity $\alpha(p)=n \geq 0$ and each $t_{i}$ is either a variable or a constant. A literal is either an atom $a$ or its negation not $a$. A (disjunctive) rule $r$ is of the form

$$
a_{1} \vee \cdots \vee a_{n}:-b_{1}, \ldots, b_{k}, \operatorname{not} b_{k+1}, \ldots, \operatorname{not} b_{m}
$$

with $n \geq 0, m \geq k \geq 0, n+m>0$, and where $a_{1}, \ldots, a_{n}, b_{1}, \ldots, b_{m}$ are atoms.

The head of $r$ is the set $H(r)=\left\{a_{1}, \ldots, a_{n}\right\}$, and the body of $r$ is the set $B(r)=$ $\left\{b_{1}, \ldots, b_{k}\right.$, not $b_{k+1}, \ldots$, not $\left.b_{m}\right\}$. Furthermore, $B^{+}(r)=\left\{b_{1}, \ldots, b_{k}\right\}$ and $B^{-}(r)=$ $\left\{b_{k+1}, \ldots, b_{m}\right\}$. We will sometimes denote a rule $r$ as $H(r):-B(r)$.

A weak constraint [8] is an expression $w c$ of the form

$$
: \sim b_{1}, \ldots, b_{k}, \operatorname{not} b_{k+1}, \ldots, \operatorname{not} b_{m} \cdot[w: l]
$$

where $m \geq k \geq 0$ and $b_{1}, \ldots, b_{m}$ are literals, while weight $(w c)=w$ (the weight) and $l$ (the level) are positive integer constants or variables. For convenience, $w$ and/or $l$ may be omitted and are set to 1 in this case. The sets $B(w c), B^{+}(w c)$, and $B^{-}(w c)$ are defined as for rules. We will sometimes denote a weak constraint $w c$ as : $\sim B(w c)$.

A program $P$ is a finite set of rules and weak constraints. Rules $(P)$ denotes the set of rules and $W C(P)$ the set of weak constraints in $P . w_{\max }^{P}$ and $l_{\max }^{P}$ denote the maximum weight and maximum level over $W C(P)$, respectively. A program (rule, atom) is propositional or ground if it does not contain variables. A program is called strong if $W C(P)=\emptyset$, and weak otherwise.

For any program $P$, let $U_{P}$ be the set of all constants appearing in $P$ (if no constant appears in $P$, an arbitrary constant is added to $U_{P}$ ); let $B_{P}$ be the set of all ground literals constructible from the predicate symbols appearing in $P$ and the constants of $U_{P}$; and let $\operatorname{Ground}(P)$ be the set of rules and weak constraints obtained by applying, to each rule and weak constraint in $P$ all possible substitutions from the variables in $P$ to elements of $U_{P} . U_{P}$ is usually called the Herbrand Universe of $P$ and $B_{P}$ the Herbrand Base of $P$.

A ground rule $r$ is satisfied by a set $I$ of ground atoms iff $H(r) \cap I \neq \emptyset$ whenever $B^{+}(r) \subseteq I$ and $B^{-}(r) \cap I=\emptyset$. $I$ satisfies a ground program $P$, if each $r \in P$ is 
satisfied by $I$. For non-ground $P, I$ satisfies $P$ iff $I$ satisfies $\operatorname{Rules}(\operatorname{Ground}(P))$. A ground weak constraint $w c$ is violated by $I$, iff $B^{+}(w c) \subseteq I$ and $B^{-}(w c) \cap I=\emptyset$; it is satisfied otherwise.

Following [13], a set $I \subseteq B_{P}$ of atoms is an answer set for a strong program $P$ iff it is a subset-minimal set that satisfies the reduct

$$
P^{I}=\left\{H(r):-B^{+}(r) \mid I \cap B^{-}(r)=\emptyset, r \in \operatorname{Ground}(P)\right\} .
$$

A set of atoms $I \subseteq B_{P}$ is an answer set for a weak program $P$ iff $I$ is an answer set of Rules $(P)$ and $H^{\text {Ground }(P)}(I)$ is minimal among all the answer sets of Rules $(P)$, where the penalization function $H^{P}(I)$ for weak constraint violation of a ground program $P$ is defined as follows:

$$
\begin{aligned}
& H^{P}(I)=\sum_{i=1}^{l_{\max }^{P}}\left(f_{P}(i) \cdot \sum_{w \in N_{i}^{P}(I)} \text { weight }(w)\right) \\
& f_{P}(1)=1, \text { and } \\
& f_{P}(n)=f_{P}(n-1) \cdot|W C(P)| \cdot w_{\max }^{P}+1 \text { for } n>1 .
\end{aligned}
$$

where $N_{i}^{P}(I)$ denotes the set of weak constraints of $P$ in level $i$ violated by $I$. For any program $P$, we denote the set of its answer sets by $A S(P)$. In this paper, we use only weak constraints with weight and level 1 , for which $H^{\operatorname{Ground}(P)}(I)$ amounts to the number of weak constraints violated in $I$.

A ground atom $a$ is a brave (sometimes also called credulous or possible) consequence of a program $P$, denoted $P \models_{b} a$, if $a \in A$ holds for at least one $A \in A S(P)$. A ground atom $a$ is a cautious (sometimes also called skeptical or certain) consequence of a program $P$, denoted $P \models_{c} a$, if $a \in A$ holds for all $A \in A S(P)$. A ground atom $a$ is a definite consequence [7] of a program $P$, denoted $P \models_{d} a$, if $A S(P) \neq \emptyset$ and $a \in A$ holds for all $A \in A S(P)$. The sets of all brave, cautious, definite consequences of a program $P$ are denoted as $B C(P), C C(P), D C(P)$, respectively.

\section{Propositional Manifold Programs}

In this section, we present a translation which essentially creates a copy of a given strong propositional program for each of (resp. for a subset of) its atoms. Thus, we require several copies of the alphabet used by the given program.

Definition 1. Given a set I of literals, a collection $\mathcal{I}$ of sets of literals, and an atom a, define $I^{a}=\left\{p^{a} \mid\right.$ atom $\left.p \in I\right\} \cup\left\{\operatorname{not} p^{a} \mid \operatorname{not} p \in I\right\}$ and $\mathcal{I}^{a}=\left\{I^{a} \mid I \in \mathcal{I}\right\}$.

The actual transformation to a manifold is given in the next definition. We copy a given program $P$ for each atom $a$ in a given set $S$, whereby the transformation guarantees the existence of an answer set by enabling the copies conditionally.

Definition 2. For a strong propositional program $P$ and $S \subseteq B_{P}$, define its manifold as

$$
P_{S}^{t r}=\bigcup_{r \in P}\left\{H(r)^{a}:-\{c\} \cup B(r)^{a} \mid a \in S\right\} \cup\{c:-\operatorname{not} i ; i:-\operatorname{not} c\} .
$$

We assume $B_{P} \cap B_{P_{S}^{t r}}=\emptyset$, that is, all symbols in $P_{S}^{t r}$ are assumed to be fresh. 
Example 1. Consider $\Phi=\{p \vee q:-; r:-p ; r:-q\}$ for which $A S(\Phi)=\{\{p, r\}$, $\{q, r\}\}, B C(\Phi)=\{p, q, r\}$ and $C C(\Phi)=D C(\Phi)=\{r\}$. When forming the manifold for $B_{\Phi}=\{p, q, r\}$, we obtain

$$
\Phi_{B_{\Phi}}^{t r}=\left\{\begin{array}{llll}
p^{p} \vee q^{p}:-c ; & r^{p}:-c, p^{p} ; r^{p}:-c, q^{p} ; c:-\operatorname{not} i ; \\
p^{q} \vee q^{q}:-c ; & r^{q}:-c, p^{q} ; r^{q}:-c, q^{q} ; i:-\operatorname{not} c ; \\
p^{r} \vee q^{r}:-c ; & r^{r}:-c, p^{r} ; r^{r}:-c, q^{r}
\end{array}\right\}
$$

Note that given a strong program $P$ and $S \subseteq B_{P}$, the construction of $P_{S}^{t r}$ can be done in polynomial time (w.r.t. the size of $P$ ). The answer sets of the transformed program consist of all combinations (of size $|S|$ ) of answer sets of the original program (augmented by $c$ ) plus the special answer set $\{i\}$ which we shall use to indicate inconsistency of $P$.

Proposition 1. For a strong propositional program $P$ and a set $S \subseteq B_{P}, A S\left(P_{S}^{t r}\right)=$ $A \cup\{\{i\}\}$, where

$$
A=\left\{\bigcup_{i=1}^{|S|} A_{i} \cup\{c\} \mid\left\langle A_{1}, \ldots, A_{|S|}\right\rangle \in \prod_{a \in S} A S(P)^{a}\right\} .
$$

Note that $\prod$ denotes the Cartesian product in Proposition 1.

Example 2. For $\Phi$ of Example 1, we obtain that $A S\left(\Phi_{B_{\Phi}}^{t r}\right)$ consists of $\{i\}$ plus (copies of $\{q, r\}$ are underlined for readability)

$$
\begin{aligned}
& \left\{c, p^{p}, r^{p}, p^{q}, r^{q}, p^{r}, r^{r}\right\},\left\{c, \underline{q^{p}, r^{p}}, p^{q}, r^{q}, p^{r}, r^{r}\right\},\left\{c, p^{p}, r^{p}, q^{q}, r^{q}, p^{r}, r^{r}\right\}, \\
& \left\{c, p^{p}, r^{p}, p^{q}, r^{q}, \underline{q}^{r}, r^{r}\right\},\left\{c,{\underline{q^{p}, r}}^{p}, \underline{q}^{q}, r^{q}, p^{r}, r^{r}\right\},\left\{c, \underline{q^{p}, r^{p}}, \bar{p}^{q}, r^{q}, \underline{q^{r}, r^{r}}\right\}, \\
& \left\{c, p^{p}, r^{p}, \underline{q}^{q}, r^{q},{\overline{q^{r}, r}}^{r}\right\},\left\{c,{\underline{q^{p}, r}}^{p},{\overline{q^{q}, r}}^{q}, \underline{q^{r}, r^{r}}\right\} \text {. }
\end{aligned}
$$

Using this transformation, each answer set encodes an association of an atom with some answer set of the original program. If an atom $a$ is a brave consequence of the original program, then a witnessing answer set exists, which contains the atom $a^{a}$. The idea is now to prefer those atom-answer set associations where the answer set is a witness. We do this by means of weak constraints and penalize each association where the atom is not in the associated answer set, that is, where $a^{a}$ is not in the answer set of the transformed program. Doing this for each atom means that an optimal answer set will not contain $a^{a}$ only if there is no answer set of the original program that contains $a$, so each $a^{a}$ contained in an optimal answer set is a brave consequence of the original program.

Definition 3. Given a strong propositional program $P$ and $S \subseteq B_{P}$, let

$$
P_{S}^{b c}=P_{S}^{t r} \cup\left\{: \sim \operatorname{not} a^{a} \mid a \in S\right\} \cup\{: \sim i\}
$$

Observe that all weak constraints are violated in the special answer set $\{i\}$, while in the answer set $\{c\}$ (which occurs if the original program has an empty answer set) all but $: \sim i$ are violated. The following result would also hold without $: \sim i$ being included. 
Proposition 2. Given a strong propositional program $P$ and $S \subseteq B_{P}$, for any $A \in$ $A S\left(P_{S}^{b c}\right),\left\{a \mid a^{a} \in A\right\}=B C(P) \cap S$.

Example 3. For the program $\Phi$ as given Example 1, $\Phi_{B_{\Phi}}^{b c}$ is given by $\Phi_{B_{\Phi}}^{t r} \cup\{: \sim$ $\left.\operatorname{not} p^{p} ;: \sim \operatorname{not} q^{q} ;: \sim \operatorname{not} r^{r} ;: \sim i\right\}$. We obtain that $A S\left(\Phi_{B_{\Phi}}^{b c}\right)=\left\{A_{1}, A_{2}\right\}$, where $A_{1}=\left\{c, p^{p}, r^{p}, q^{q}, r^{q}, p^{r}, r^{r}\right\}$ and $A_{2}=\left\{c, p^{p}, r^{p}, q^{q}, r^{q}, q^{r}, r^{r}\right\}$, as these two answer sets are the only ones that violate no weak constraint. We can observe that $\left\{a \mid a^{a} \in A_{1}\right\}=\left\{a \mid a^{a} \in A_{2}\right\}=\{p, q, r\}=B C(\Phi)$.

Concerning cautious consequences, we first observe that if a program is inconsistent (in the sense that it does not have any answer set), each atom is a cautious consequence. But if $P$ is inconsistent, then $P_{S}^{t r}$ will have only $\{i\}$ as an answer set, so we will need to find a suitable modification in order to deal with this in the correct way. In fact, we can use a similar approach as for brave consequences, but penalize those associations where an atom is contained in its associated answer set. Any optimal answer set will thus contain $a^{a}$ for an atom only if $a$ is contained in each answer set. If an answer set containing $i$ exists, it is augmented by all atoms $a^{a}$, which also causes all weak constraints to be violated.

Definition 4. Given a strong propositional program $P$ and $S \subseteq B_{P}$, let

$$
P_{S}^{c c}=P_{S}^{t r} \cup\left\{: \sim a^{a} \mid a \in S\right\} \cup\left\{a^{a}:-i \mid a \in S\right\} \cup\{: \sim i\}
$$

As for $P_{S}^{b c}$, the following result also holds without including $: \sim i$.

Proposition 3. Given a strong propositional program $P$ and $S \subseteq B_{P}$, for any $A \in$ $A S\left(P_{S}^{c c}\right),\left\{a \mid a^{a} \in A\right\}=C C(P) \cap S$.

Example 4. Recall program $\Phi$ from Example 1. We have $\Phi_{B_{\Phi}}^{c c}=\Phi_{B_{\Phi}}^{t r} \cup\left\{: \sim p^{p} ;: \sim\right.$ $\left.q^{q} ;: \sim r^{r} ; p^{p}:-i ; q^{q}:-i ; r^{r}:-i ;: \sim i\right\}$. We obtain that $A S\left(\Phi_{B_{\Phi}}^{c c}\right)=\left\{A_{3}, A_{4}\right\}$, where $A_{3}=\left\{c, q^{p}, r^{p}, p^{q}, r^{q}, p^{r}, r^{r}\right\}$ and $A_{4}=\left\{c, q^{p}, r^{p}, p^{q}, r^{q}, q^{r}, r^{r}\right\}$, as these two answer sets are the only ones that violate only one weak constraint, namely : $\sim r^{r}$. We observe that $\left\{a \mid a^{a} \in A_{3}\right\}=\left\{a \mid a^{a} \in A_{4}\right\}=\{r\}=C C(\Phi)$.

We next consider the notion of definite consequences. Different to cautious consequences, we do not add the annotated atoms to the answer set containing $i$. However, this answer set should never be among the optimal ones unless it is the only one. Therefore we inflate it by new atoms $i^{a}$, all of which incur a penalty. This guarantees that this answer set will incur a higher penalty $\left(\left|B_{P}\right|+1\right)$ than any other $\left(\leq\left|B_{P}\right|\right)$.

Definition 5. Given a strong propositional program $P$ and $S \subseteq B_{P}$, let

$$
P_{S}^{d c}=P_{S}^{t r} \cup\left\{: \sim a^{a} ; i^{a}:-i ;: \sim i^{a} \mid a \in S\right\} \cup\{: \sim i\}
$$

Proposition 4. Given a strong propositional program $P$ and $S \subseteq B_{P}$, for any $A \in$ $A S\left(P_{S}^{d c}\right),\left\{a \mid a^{a} \in A\right\}=D C(P) \cap S$.

Example 5. Recall program $\Phi$ from Example 1. We have $\Phi_{B_{\Phi}}^{d c}=\Phi_{B_{\Phi}}^{t r} \cup\left\{: \sim p^{p} ;: \sim \sim\right.$ $\left.q^{q} ;: \sim r^{r} ; i^{p}:-i ; i^{q}:-i ; i^{r}:-i: \sim i^{p} ;: \sim i^{q} ;: \sim i^{r} ;: \sim i\right\}$. As in Example $4, A_{3}$ and $A_{4}$ are the only ones that violate only one weak constraint, namely $: \sim r^{r}$, and thus are the answer sets of $\Phi_{B_{\Phi}}^{d c}$. 
Obviously, one can compute all brave, cautious, or definite consequences of a program by choosing $S=B_{P}$. We also note that the programs from Definitions 3, 4 and 5 yield multiple answer sets. However each of these yields the same atoms $a^{a}$, so it is sufficient to compute one of these. The programs could be extended in order to admit only one answer set by suitably penalizing all atoms $a^{b}(a \neq b)$. To avoid interference with the weak constraints already used, these additional weak constraints would have to pertain to a different level.

\section{Non-Ground Manifold Programs}

We now generalize the techniques introduced in Section 3 to non-ground strong programs. In principle, one could annotate each predicate (rather than atom as in Section 3) with ground atoms of a subset of the Herbrand Base. However, one can also move the annotations to the non-ground level: For example, instead of annotating a rule $p(X, Y):-q(X, Y)$ by the set $\{r(a), r(b)\}$ yielding $p^{r(a)}(X, Y):-q^{r(a)}(X, Y)$ and $p^{r(b)}(X, Y):-q^{r(b)}(X, Y)$ we will annotate using only the predicate $r$ and extend the arguments of $p$, yielding the compact rule $\mathrm{d}_{p}^{r}(X, Y, Z):-\mathrm{d}_{q}^{r}(X, Y, Z)$ (we use predicate symbols $\mathrm{d}_{p}^{r}$ and $\mathrm{d}_{q}^{r}$ rather than $p^{r}$ and $q^{r}$ just for pointing out the difference between annotation by predicates versus annotation by ground atoms). In this particular example we have assumed that the program is to be annotated by all ground instances of $r(Z)$; we will use this assumption also in the following for simplifying the presentation. In practice, one can clearly add atoms to the rule body for restricting the instances of the predicate by which we annotate, in the example this would yield $p^{r}(X, Y, Z):-q^{r}(X, Y, Z), \operatorname{dom}(Z)$ where the predicate $d o m$ should be defined appropriately. In the following, recall that $\alpha(p)$ denotes the arity of a predicate $p$.

Definition 6. Given an atom $a=p\left(t_{1}, \ldots, t_{n}\right)$ and a predicate $q$, let $a_{q}^{\text {tr }}$ be the atom $\mathrm{d}_{p}^{q}\left(t_{1}, \ldots, t_{n}, X_{1}, \ldots, X_{\alpha(q)}\right)$ where $X_{1}, \ldots, X_{\alpha(q)}$ are fresh variables and $\mathrm{d}_{p}^{q}$ is a new predicate symbol with $\alpha\left(\mathrm{d}_{p}^{q}\right)=\alpha(p)+\alpha(q)$. Furthermore, given a set $\mathcal{L}$ of literals, and a predicate $q$, let $\mathcal{L}_{q}^{\text {tr }}$ be $\left\{a_{q}^{\text {tr }} \mid\right.$ atom $\left.a \in \mathcal{L}\right\} \cup\left\{\operatorname{not} a_{q}^{\text {tr }} \mid \operatorname{not} a \in \mathcal{L}\right\}$.

Note that we assume that even though the variables $X_{1}, \ldots, X_{\alpha(q)}$ are fresh, they will be the same for each $a_{q}^{t r}$. One could define similar notions also for partially ground atoms or for sets of atoms characterized by a collection of defining rules, from which we refrain here for the ease of presentation. We define the manifold program in analogy to Definition 2, the only difference being the different way of annotating.

Definition 7. Given a strong program $P$ and a set $S$ of predicates, define its manifold as

$$
P_{S}^{t r}=\bigcup_{r \in P}\left\{H(r)_{q}^{t r}:-\{c\} \cup B(r)_{q}^{t r} \mid q \in S\right\} \cup\{c:-\operatorname{not} i ; i:-\operatorname{not} c\} .
$$

Example 6. Consider program $\Psi=\{p(X) \vee q(X):-r(X) ; \quad ; r(a):-\quad ; r(b):-\}$ for which $A S(\Psi)=\{\{p(a), p(b), r(a), r(b)\},\{p(a), q(b), r(a), r(b)\},\{q(a), p(b), r(a)$, $r(b)\},\{q(a), q(b), r(a), r(b)\}\}$. Hence, $B C(\Psi)=\{p(a), p(b), q(a), q(b), r(a), r(b)\}$ and $C C(\Psi)=D C(\Psi)=\{r(a), r(b)\}$. Forming the manifold for $S=\{p\}$, we obtain

$$
\Psi_{S}^{t r}=\left\{\begin{array}{l}
\mathrm{d}_{p}^{p}\left(X, X_{1}\right) \vee \mathrm{d}_{q}^{p}\left(X, X_{1}\right):-\mathrm{d}_{r}^{p}\left(X, X_{1}\right), c ; \\
\mathrm{d}_{r}^{p}\left(a, X_{1}\right):-c ; \mathrm{d}_{r}^{p}\left(b, X_{1}\right):-c ; c:-\operatorname{not} i ; i:-\operatorname{not} c
\end{array}\right\}
$$


$A S\left(\Psi_{S}^{t r}\right)$ consists of $\{i\}$ plus 16 answer sets, corresponding to all combinations of the 4 answer sets in $A S(\Psi)$.

Now we are able to generalize the encodings for brave, cautious, and definite consequences. These definitions are direct extensions of Definitions 3, 4, and 5, the differences are only due to the non-ground annotations. In particular, the diagonalization atoms $a^{a}$ should now be written as $\mathrm{d}_{p}^{p}\left(X_{1}, \ldots, X_{\alpha(p)}, X_{1}, \ldots, X_{\alpha(p)}\right)$ which represent the set of ground instances of $p\left(X_{1}, \ldots, X_{\alpha(p)}\right)$, each annotated by itself. So, a weak constraint : $\mathrm{d}_{p}^{p}\left(X_{1}, \ldots, X_{\alpha(p)}, X_{1}, \ldots, X_{\alpha(p)}\right)$ gives rise to $\left\{: \sim \mathrm{d}_{p}^{p}\left(c_{1}, \ldots, c_{\alpha(p)}\right.\right.$, $\left.\left.c_{1}, \ldots, c_{\alpha(p)}\right) \mid c_{1}, \ldots, c_{\alpha(p)} \in U\right\}$ where $U$ is the Herbrand base of the program in question, that is one weak constraint for each ground instance annotated by itself.

Definition 8. Given a strong program $P$ and a set $S$ of predicate symbols, let

$$
\begin{aligned}
& P_{S}^{b c}=P_{S}^{t r} \cup\left\{: \sim \operatorname{not} \Delta_{q} \mid q \in S\right\} \cup\{: \sim i\} \\
& P_{S}^{c c}=P_{S}^{t r} \cup\left\{: \sim \Delta_{q} ; \Delta_{q}:-i \mid q \in S\right\} \cup\{: \sim i\} \\
& P_{S}^{d c}=P_{S}^{t r} \cup\left\{: \sim \Delta_{q} ; I_{q}:-i ;: \sim I_{q} \mid q \in S\right\} \cup\{: \sim i\}
\end{aligned}
$$

where $\Delta_{q}=\mathrm{d}_{q}^{q}\left(X_{1}, \ldots, X_{\alpha(q)}, X_{1}, \ldots, X_{\alpha(q)}\right)$ and $I_{q}=i_{q}\left(X_{1}, \ldots, X_{\alpha(q)}\right)$.

Proposition 5. Given a strong program $P$ and a set $S$ of predicates, for an arbitrary $A \in A S\left(P_{S}^{b c}\right)$, (resp., $\left.A \in A S\left(P_{S}^{c c}\right), A \in A S\left(P_{S}^{d c}\right)\right)$, the set $\left\{p\left(c_{1}, \ldots, c_{\alpha(p)}\right)\right.$ $\left.\mathrm{d}_{p}^{p}\left(c_{1}, \ldots, c_{\alpha(p)}, c_{1}, \ldots, c_{\alpha(p)}\right) \in A\right\}$ is the set of brave (resp., cautious, definite) consequences of $P$ with a predicate in $S$.

Example 7. Consider again $\Psi$ and $S=\{p\}$ from Example 6. We obtain $\Psi_{S}^{b c}=\Psi_{S}^{t r} \cup$ $\left\{: \sim \operatorname{not} \mathrm{d}_{p}^{p}\left(X_{1}, X_{1}\right) ;: \sim i\right\}$ and we can check that $A S\left(\Psi_{S}^{b c}\right)$ consists of the sets

$R \cup\left\{\mathrm{d}_{p}^{p}(a, a), \mathrm{d}_{p}^{p}(b, b), \mathrm{d}_{q}^{p}(a, b), \mathrm{d}_{q}^{p}(b, a)\right\}, R \cup\left\{\mathrm{d}_{p}^{p}(a, a), \mathrm{d}_{p}^{p}(b, b), \mathrm{d}_{p}^{p}(a, b), \mathrm{d}_{q}^{p}(b, a)\right\}$, $R \cup\left\{\mathrm{d}_{p}^{p}(a, a), \mathrm{d}_{p}^{p}(b, b), \mathrm{d}_{q}^{p}(a, b), \mathrm{d}_{p}^{p}(b, a)\right\}, R \cup\left\{\mathrm{d}_{p}^{p}(a, a), \mathrm{d}_{p}^{p}(b, b), \mathrm{d}_{p}^{p}(b, a), \mathrm{d}_{p}^{p}(b, a)\right\} ;$

where $R=\left\{\mathrm{d}_{r}^{p}(a, a), \mathrm{d}_{r}^{p}(a, b), \mathrm{d}_{r}^{p}(b, a), \mathrm{d}_{r}^{p}(b, b)\right\}$. For each $A$ of these answer sets we obtain $\left\{p(t) \mid \mathrm{d}_{p}^{p}(t, t) \in A\right\}=\{p(a), p(b)\}$ which corresponds exactly to the brave consequences of $\Psi$ with a predicate of $S=\{p\}$.

For cautious consequences, $\Psi_{S}^{c c}=\Psi_{S}^{t r} \cup\left\{: \sim \mathrm{d}_{p}^{p}\left(X_{1}, X_{1}\right) ; \mathrm{d}_{p}^{p}\left(X_{1}, X_{1}\right):-i ; \quad: \sim i\right\}$ and we can check that $A S\left(\Psi_{S}^{c c}\right)$ consists of the sets

$$
\begin{aligned}
& R \cup\left\{\mathrm{d}_{q}^{p}(a, a), \mathrm{d}_{q}^{p}(b, b), \mathrm{d}_{q}^{p}(a, b), \mathrm{d}_{q}^{p}(b, a)\right\}, R \cup\left\{\mathrm{d}_{q}^{p}(a, a), \mathrm{d}_{q}^{p}(b, b), \mathrm{d}_{p}^{p}(a, b), \mathrm{d}_{q}^{p}(b, a)\right\}, \\
& R \cup\left\{\mathrm{d}_{q}^{p}(a, a), \mathrm{d}_{q}^{p}(b, b), \mathrm{d}_{q}^{p}(a, b), \mathrm{d}_{p}^{p}(b, a)\right\}, R \cup\left\{\mathrm{d}_{q}^{p}(a, a), \mathrm{d}_{q}^{p}(b, b), \mathrm{d}_{p}^{p}(b, a), \mathrm{d}_{p}^{p}(b, a)\right\} ;
\end{aligned}
$$

where $R=\left\{\mathrm{d}_{r}^{p}(a, a), \mathrm{d}_{r}^{p}(a, b), \mathrm{d}_{r}^{p}(b, a), \mathrm{d}_{r}^{p}(b, b)\right\}$. For each $A$ of these answer sets we obtain $\left\{p(t) \mid \mathrm{d}_{p}^{p}(t, t) \in A\right\}=\emptyset$ and indeed there are no cautious consequences of $\Psi$ with a predicate of $S=\{p\}$.

Finally, for definite consequences, $\Psi_{S}^{d c}=\Psi_{S}^{t r} \cup\left\{: \sim \mathrm{d}_{p}^{p}\left(X_{1}, X_{1}\right) ; i_{p}\left(X_{1}\right):-i \quad ; \quad: \sim\right.$ $\left.i_{p}\left(X_{1}\right) ;: \sim i\right\}$. It is easy to see that $A S\left(\Psi_{S}^{d c}\right)=A S\left(\Psi_{S}^{c c}\right)$ and so $\left\{p(t) \mid \mathrm{d}_{p}^{p}(t, t) \in\right.$ $A\}=\emptyset$ for each answer set $A$ of $\Psi_{S}^{d c}$, and indeed there is also no definite consequence of $\Psi$ with a predicate of $S=\{p\}$. 
These definitions exploit the fact that the semantics of non-ground programs is defined via their grounding with respect to their Herbrand Universe. So the fresh variables introduced in the manifold will give rise to one copy of a rule for each ground atom.

In practice, ASP systems usually require rules to be safe, that is, that each variable occurs (also) in the positive body. The manifold for a set of predicates may therefore contain unsafe rules (because of the fresh variables). But this can be repaired by adding a domain atom $\operatorname{dom}_{q}\left(X_{1}, \ldots, X_{m}\right)$ to a rule which is to be annotated with $q$. This predicate can in turn be defined by a rule $\operatorname{dom}_{q}\left(X_{1}, \ldots, X_{m}\right):-u\left(X_{1}\right), \ldots, u\left(X_{m}\right)$ where $u$ is defined using $\left\{u(c) \mid c \in U_{P}\right\}$. One can also provide smarter definitions for $d o m_{q}$ by using a relaxation of the definition for $q$.

We also observe that ground atoms that are contained in all answer sets of a program need not be annotated in the manifold. Note that these are essentially the cautious consequences of a program and therefore determining all of those automatically before rewriting does not make sense. But for some atoms this property can be determined only by the structure of the program. For instance, facts will be in all answer sets. In the sequel we will not annotate extensional atoms (those defined only by facts) in order to obtain more concise programs. One could also go further and omit the annotation of atoms which are defined using nondisjunctive stratified programs.

As an example, we present an ASP encoding for boolean satisfiability and then create its manifold program for resolving the following problem: Given a propositional formula in $\operatorname{CNF} \varphi$, compute all atoms which are true in all models of $\varphi$. We provide a fixed program which takes a representation of $\varphi$ as facts as input. To apply our method we first require a program whose answer sets are in a one-to-one correspondence to the models of $\varphi$. To start with, we fix the representation of CNFs. Let $\varphi$ (over atoms $A$ ) be of the form $\bigwedge_{i=1}^{n} c_{i}$. Then, $D_{\varphi}=\{\operatorname{at}(a) \mid a \in A\} \cup\{\operatorname{cl}(i) \mid 1 \leq i \leq n\} \cup\{\operatorname{pos}(a, i) \mid$ atom $a$ occurs positively in $\left.c_{i}\right\} \cup\left\{\operatorname{neg}(a, i) \mid\right.$ atom $a$ occurs negatively in $\left.c_{i}\right\}$. We construct program SAT as the set of the following rules.

$$
\begin{aligned}
& \operatorname{true}(X):-\operatorname{not} f a l s e(X), \operatorname{at}(X) ; \quad f a l s e(X):-\operatorname{not} \operatorname{true}(X), \operatorname{at}(X) ; \\
& \text { ok }(C):-\operatorname{true}(X), \operatorname{pos}(C, X) ; \text { ok }(C):- \text { false }(X), \operatorname{neg}(C, X) ;:-\operatorname{not} \text { ok }(C), \operatorname{cl}(C) .
\end{aligned}
$$

It can be checked that the answer sets of $\operatorname{SAT} \cup D_{\varphi}$ are in a one-to-one correspondence to the models (over $A$ ) of $\varphi$. In particular, for any model $I \subseteq A$ of $\varphi$ there exists an answer set $M$ of SAT $\cup D_{\varphi}$ such that $I=\{a \mid \operatorname{true}(a) \in M\}$. We now consider $\mathrm{SAT}_{\{\text {true }\}}^{c c}$ which consists of the following rules.

$$
\begin{array}{rll}
\mathrm{d}_{\text {true }}^{\text {true }}(X, Y):-c, \operatorname{not} \mathrm{d}_{\text {false }}^{\text {true }}(X, Y), \operatorname{at}(X) ; c:-\operatorname{not} i ; & i:-\operatorname{not} c ; \\
\mathrm{d}_{\text {false }}^{\text {true }}(X, Y):-c, \operatorname{not} \mathrm{d}_{\text {true }}^{\text {true }}(X, Y), \operatorname{at}(X) ; & :-c, \operatorname{not} \mathrm{d}_{\text {ok }}^{\text {true }}(C, Y), \operatorname{cl}(C) ; \\
\mathrm{d}_{\text {ok }}^{\text {true }}(C, Y):-c, \mathrm{~d}_{\text {true }}^{\text {true }}(X, Y), \operatorname{pos}(C, X) ; & : \sim \mathrm{d}_{\text {true }}^{\text {true }}(X, X) ; \quad: \sim i ; \\
\mathrm{d}_{\text {ok }}^{\text {true }}(C, Y):-c, \mathrm{~d}_{\text {false }}^{\text {true }}(X, Y), \operatorname{neg}(C, X) ; & \mathrm{d}_{\text {true }}^{\text {true }}(X, X):-i .
\end{array}
$$

Given Proposition 5, it is easy to see that, given some answer set $A$ of $\operatorname{SAT}_{\{\text {true }}^{c c} \cup D_{\varphi}$, $\left\{a \mid \mathrm{d}_{\text {true }}^{\text {true }}(a, a) \in A\right\}$ is precisely the set of atoms which are true in all models of $\varphi$.

\section{Applications}

In this section, we put our technique to work and show how to use meta-reasoning over answer sets for two application scenarios. The first one is a well-known problem from 
propositional logic, and we will reuse the example from above. The second example takes a bit more background, but presents a novel method to compute ideal extensions for argumentation frameworks.

\subsection{The Unique Minimal Model Problem}

As a first example, we show how to encode the problem of deciding whether a given propositional formula $\varphi$ has a unique minimal model. This problem is known to be in $\Theta_{2}^{P}$ and to be co-NP-hard (the exact complexity is an open problem). Let $I$ be the intersection of all models of $\varphi$. Then $\varphi$ has a unique minimal model iff $I$ is also a model of $\varphi$. We thus use our example from the previous section, and define the program UNIQUE as $\mathrm{SAT}_{\{\text {true }\}}^{c c}$ augmented by rules $o k(C):-\mathrm{d}_{\text {true }}^{\text {true }}(X, X), \operatorname{pos}(C, X)$; $o k(C):-\operatorname{not} \mathrm{d}_{\text {true }}^{\text {true }}(X, X), \operatorname{neg}(C, X)$; :- not ok $(C), \operatorname{cl}(C)$.

Theorem 1. For any CNF formula $\varphi$, it holds that $\varphi$ has a unique minimal model, if and only if program UNIQUE $\cup D_{\varphi}$ has at least one answer set.

A slight adaption of this encoding allows us to formalize CWA-reasoning [10] over a propositional knowledge base $\varphi$, since the atoms $a$ in $\varphi$, for which the corresponding atoms $\mathrm{d}_{\text {true }}^{\text {true }}(a, a)$ are not contained in an answer set of $\operatorname{SAT}_{\{\text {true }}^{c c} \cup D_{\varphi}$, are exactly those which are added negated to $\varphi$ for CWA-reasoning.

\subsection{Computing the Ideal Extension}

Our second example is from the area of argumentation, where the problem of computing the ideal extension [12] of an abstract argumentation framework was recently shown to be complete for $\mathrm{FP}_{\|}^{\mathrm{NP}}$ in [14]. Thus, this task cannot be compactly encoded via normal programs (under usual complexity theoretic assumptions). On the other hand, the complexity shows that employing disjunction is not necessary, if one instead uses weak constraints. We first give the basic definitions following [15].

Definition 9. $A n$ argumentation framework (AF) is a pair $F=(A, R)$ where $A \subseteq \mathcal{U}$ is a set of arguments and $R \subseteq A \times A$. $(a, b) \in R$ means that a attacks $b$. An argument $a \in A$ is defended by $S \subseteq A$ (in $F$ ) if, for each $b \in A$ such that $(b, a) \in R$, there exists a $c \in S$, such that $(c, b) \in R$. An argument a is admissible (in $F$ ) w.r.t. a set $S \subseteq A$ if each $b \in A$ which attacks $a$ is defended by $S$.

Semantics for argumentation frameworks are given in terms of so-called extensions. The next definitions introduce two such notions which also underly the concept of an ideal extension.

Definition 10. Let $F=(A, R)$ be an $A F$. A set $S \subseteq A$ is said to be conflict-free (in $F)$, if there are no $a, b \in S$, such that $(a, b) \in R$. A set $S$ is an admissible extension of $F$, if $S$ is conflict-free in $F$ and each $a \in S$ is admissible in $F$ w.r.t. S. The collection of admissible extensions is denoted by adm $(F)$. An admissible extension $S$ of $F$ is a preferred extension of $F$, if for each $T \in \operatorname{adm}(F), S \not \subset T$. The collection of preferred extensions of $F$ is denoted by pref $(F)$. 
Definition 11. Let $F$ be an AF. A set $S$ is called ideal for $F$, if $S \in \operatorname{adm}(F)$ and $S \subseteq \bigcap_{T \in \operatorname{pref}(F)}$ T. A maximal (w.r.t. set-inclusion) ideal set of $F$ is called an ideal extension of $F$.

It was shown that for each AF $F$, a unique ideal extension exists. In [14], the following algorithm to compute the ideal extension of an $\operatorname{AF} F=(A, R)$ is proposed. Let $X_{F}^{-}=A \backslash \bigcup_{S \in \operatorname{adm}(F)} S$ and $X_{F}^{+}=\{a \in A \mid \forall b, c:(b, a),(a, c) \in R \Rightarrow b, c \in$ $\left.X_{F}^{-}\right\} \backslash X_{F}^{-}$, and define an $\mathrm{AF} F^{*}=\left(X_{F}^{+} \cup X_{F}^{-}, R^{*}\right)$ where $R^{*}=R \cap\{(a, b),(b, a) \mid$ $\left.a \in X_{F}^{+}, b \in X_{F}^{-}\right\} . F^{*}$ is a bipartite $\mathrm{AF}$ in the sense that $R^{*}$ is a bipartite graph.

Proposition 6 ([14]). The ideal extension of $A F F$ is given by $\bigcup_{S \in \operatorname{adm}\left(F^{*}\right)}\left(S \cap X_{F}^{+}\right)$.

The set of all admissible atoms for a bipartite AF $F$ can be computed in polynomial time using Algorithm 1 of [16]. This is basically a fixpoint iteration identifying arguments in $X_{F}^{+}$that cannot be in an admissible extension: First, arguments in $X_{0}=X_{F}^{+}$ are excluded, which are attacked by unattacked arguments (which are necessarily in $X_{F}^{-}$), yielding $X_{1}$. Now, arguments in $X_{F}^{-}$may be unattacked by $X_{1}$, and all arguments in $X_{1}$ attacked by such newly unattacked arguments should be excluded. This process is iterated until either no arguments are left or no more argument can be excluded. There may be at most $\left|X_{F}^{+}\right|$iterations in this process.

We exploit this technique to formulate an ASP-encoding IDEAL. We first report a program the answer sets of which characterize admissible extensions. Then, we use the brave manifold of this program in order to determine all arguments contained in some admissible extension. Finally, we extend this manifold program in order to identify $F^{*}$ and to simulate Algorithm 1 of [16].

The argumentation frameworks will be given to IDEAL as sets of input facts. Given an $\mathrm{AF} F=(A, R)$, let $D_{F}=\{a(x) \mid x \in A\} \cup\{\mathrm{r}(x, y) \mid(x, y) \in R\}$. Program ADM, given by the rules below, computes admissible extensions (cf. [17, 18]):

$$
\begin{aligned}
& \operatorname{in}(X):-\operatorname{not} \operatorname{out}(X), a(X) ; \operatorname{out}(X):-\operatorname{not} \operatorname{in}(X), a(X) ; \operatorname{def}(X):-\operatorname{in}(Y), \mathrm{r}(Y, X) ; \\
& :-\operatorname{in}(X), \operatorname{in}(Y), \mathrm{r}(X, Y) ;:-\operatorname{in}(X), \mathrm{r}(Y, X), \operatorname{not} \operatorname{def}(Y) .
\end{aligned}
$$

Indeed one can show that, given an $\mathrm{AF} F$, the answer sets of $\mathrm{ADM} \cup D_{F}$ are in a one-to-one correspondence to the admissible extensions of $F$ via the in $(\cdot)$ predicate. In order to determine the brave consequences of ADM for predicate in, we form $\mathrm{ADM}_{\{\text {in }\}}^{b c}$, and extend it by collecting all brave consequences of $\mathrm{ADM} \cup D_{F}$ in predicate in $(\cdot)$, from which we can determine $X_{F}^{-}$(represented by in $\left.{ }^{-}(\cdot)\right), X_{F}^{+}$(represented by in ${ }^{+}(\cdot)$, using auxiliary predicate not_in ${ }^{+}(\cdot)$ ), and $R^{*}$ (represented by $\mathrm{q}(\cdot, \cdot)$ ).

$$
\begin{array}{ll}
\operatorname{in}(X):-\mathrm{d}_{\mathrm{in}}^{\mathrm{in}}(X, X) ; \mathrm{in}^{-}(X):-a(X), \operatorname{not} \operatorname{in}(X) ; \operatorname{in}^{+}(X):-\operatorname{in}(X), \text { not not_in } \\
\text { not_in }^{+}(X):-\operatorname{in}(Y), r(X, Y) ; & \text { not_in }^{+}(X):-\operatorname{in}(Y), r(Y, X) ; \\
\mathrm{q}(X, Y):-\mathrm{r}(X, Y), \mathrm{in}^{+}(X), \mathrm{in}^{-}(Y) ; & \mathrm{q}(X, Y):-\mathrm{r}(X, Y), \mathrm{in}^{-}(X), \mathrm{in}^{+}(Y) .
\end{array}
$$

In order to simulate Algorithm 1 of [16], we use the elements in $X_{F}^{+}$for marking the iteration steps. To this end, we use an arbitrary order $<$ on ASP constants (all ASP systems provide such a predefined order) and define successor, infimum and supremum among the constants representing $X_{F}^{+}$w.r.t. the order $<$. 


$$
\begin{aligned}
\operatorname{nsucc}(X, Z) & :-\operatorname{in}^{+}(X), \operatorname{in}^{+}(Y), \operatorname{in}^{+}(Z), X<Y, Y<Z ; \\
\operatorname{succ}(X, Y) & :-\operatorname{in}^{+}(X), \operatorname{in}^{+}(Y), X<Y, \operatorname{not} \operatorname{nsucc}(X, Y) ; \\
\operatorname{ninf}(Y) & :-\operatorname{in}^{+}(X), \operatorname{in}^{+}(Y), X<Y ; \operatorname{nsup}(X):-\operatorname{in}^{+}(X), \operatorname{in}^{+}(Y), X<Y ; \\
\inf (X) & :-\operatorname{in}^{+}(X), \operatorname{not} \operatorname{ninf}(X) ; \quad \sup (X):-\operatorname{in}^{+}(X), \operatorname{not} \operatorname{nsup}(X) .
\end{aligned}
$$

We now use this to iteratively determine arguments that are not in the ideal extension, using $\operatorname{nid}(\cdot, \cdot)$, where the first argument is the iteration step. In the first iteration (identified by the infimum) all arguments in $X_{F}^{+}$which are attacked by an unattacked argument are collected. In subsequent iterations, all arguments from the previous steps are included and augmented by arguments that are attacked by an argument not attacked by arguments in $X_{F}^{+}$that were not yet excluded in the previous iteration. Finally, arguments in the ideal extension are those that are not excluded from $X_{F}^{+}$in the final iteration (identified by the supremum).

$$
\begin{aligned}
\operatorname{att}_{0}(X) & :-\mathrm{q}(Y, X) ; \quad \operatorname{att}_{i}(J, Z):-q(Y, Z), \operatorname{in}^{+}(Y), \operatorname{not} \operatorname{nid}(J, Y), \operatorname{in}^{+}(J) ; \\
\operatorname{ideal}(X) & :-\operatorname{in}^{+}(X), \sup (I), \operatorname{not} \operatorname{nid}(I, X) ; \operatorname{nid}(I, Y):-\operatorname{succ}(J, I), \operatorname{nid}(J, Y) ; \\
\operatorname{nid}(I, Y) & :-\inf (I), \mathrm{q}(Z, Y), \operatorname{in}^{+}(Y), \operatorname{not} \operatorname{att}_{0}(Z) ; \\
\operatorname{nid}(I, Y) & :-\operatorname{succ}(J, I), q(Z, Y), \operatorname{in}^{+}(Y), \operatorname{not} \operatorname{att}_{i}(J, Z) .
\end{aligned}
$$

If we put $\mathrm{ADM}_{\{\mathrm{in}\}}^{b c}$ and all of these additional rules together to form the program IDEAL, we obtain the following result:

Theorem 2. Let $F$ be an $A F$ and $A \in A S\left(\right.$ IDEAL $\left.\cup D_{F}\right)$. Then, the ideal extension of $F$ is given by $\{a \mid \operatorname{ideal}(a) \in A\}$.

\section{Conclusion}

In this paper, we provided a novel method to rewrite ASP-programs in such a way that reasoning over all answer sets of the original program can be formulated within the same program. Our method exploits the well-known concept of weak constraints. We illustrated the impact of our method by encoding the problems of (i) deciding whether a propositional formula in CNF has a unique minimal model, and (ii) computing the ideal extension of an argumentation framework. Known complexity results witness that our encodings are adequate in the sense that efficient ASP encodings without weak constraints or similar constructs are assumed to be infeasible.

The manifold program for cautious consequences is also closely related to the concept of data disjunctions [19] (this paper also contains a detailed discussion about the complexity class $\Theta_{2}^{P}$ and related classes for functional problems). Related work has also been done in the area of default logic, where a method for reasoning within a single extension has been proposed [20]. That method uses set-variables which characterize the set of generating defaults of the original extensions. Such an approach differs considerably from ours as it encodes certain aspects of the semantics (which ours does not), which puts it closer to meta-programming (cf. [21]). 
As future work, we are interested in developing a suitable language for expressing reasoning with brave, cautious and definite consequences, allowing also for mixing different reasoning modes. This language should serve as a platform for natural encodings of problems in complexity classes $\Theta_{2}^{P}, \Theta_{3}^{P}, \mathrm{FP}_{\|}^{\mathrm{NP}}$, and $\mathrm{FP}_{\|}^{\Sigma_{2}^{P}}$. Moreover, we intend studying the use of alternative preferential constructs in place of weak constraints.

\section{References}

1. Marek, V.W., Truszczyński, M.: Stable models and an alternative logic programming paradigm. In: The Logic Programming Paradigm - A 25-Year Perspective. (1999) 375398

2. Niemelä, I.: Logic programming with stable model semantics as a constraint programming paradigm. Ann. Math. Artif. Intell. 25(3-4) (1999) 241-273

3. Baral, C.: Knowledge Representation, Reasoning and Declarative Problem Solving. CUP (2002)

4. Gelfond, M.: Representing knowledge in A-Prolog. In: Computational Logic: From Logic Programming into the Future. LNCS 2408, (2002) 413-451

5. Gebser, M., Liu, L., Namasivayam, G., Neumann, A., Schaub, T., Truszczyński, M.: The first answer set programming system competition. In: LPNMR'07. LNCS 4483, (2007) 3-17

6. Bravo, L., Bertossi, L.E.: Logic programs for consistently querying data integration systems. In: IJCAI 2003,(2003) 10-15

7. Saccà, D.: Multiple total stable models are definitely needed to solve unique solution problems. Inf. Process. Lett. 58(5) (1996) 249-254

8. Buccafurri, F., Leone, N., Rullo, P.: Enhancing disjunctive datalog by constraints. IEEE Trans. Knowl. Data Eng. 12(5) (2000) 845-860

9. Leone, N., Pfeifer, G., Faber, W., Eiter, T., Gottlob, G., Perri, S., Scarcello, F.: The dlv system for knowledge representation and reasoning. ACM Trans. Comput. Log. 7(3) (2006) 499-562

10. Reiter, R.: On closed world data bases. In: Logic and Databases. Plenum Press (1978) 55-76

11. Bench-Capon, T.J.M., Dunne, P.E.: Argumentation in artificial intelligence. Artif. Intell. 171(10-15) (2007) 619-641

12. Dung, P.M., Mancarella, P., Toni, F.: Computing ideal sceptical argumentation. Artif. Intell. 171(10-15) (2007) 642-674

13. Gelfond, M., Lifschitz, V.: Classical negation in logic programs and disjunctive databases. New Generation Comput. 9(3/4) (1991) 365-386

14. Dunne, P.E.: The computational complexity of ideal semantics I: Abstract argumentation frameworks. In: COMMA'08, IOS Press (2008) 147-158

15. Dung, P.M.: On the acceptability of arguments and its fundamental role in nonmonotonic reasoning, logic programming and n-person games. Artif. Intell. 77(2) (1995) 321-358

16. Dunne, P.E.: Computational properties of argument systems satisfying graph-theoretic constraints. Artif. Intell. 171(10-15) (2007) 701-729

17. Osorio, M., Zepeda, C., Nieves, J.C., Cortés, U.: Inferring acceptable arguments with answer set programming. In: ENC 2005 (2005) 198-205

18. Egly, U., Gaggl, S., Woltran, S.: Answer-set programming encodings for argumentation frameworks. In: Proceedings ASPOCP'08. (2008)

19. Eiter, T., Veith, H.: On the complexity of data disjunctions. Theor. Comput. Sci. 288(1) (2002) 101-128

20. Delgrande, J.P., Schaub, T.: Reasoning credulously and skeptically within a single extension. Journal of Applied Non-Classical Logics 12(2) (2002) 259-285

21. Eiter, T., Faber, W., Leone, N., Pfeifer, G.: Computing preferred answer sets by metainterpretation in answer set programming. TPLP 3(4-5) (2003) 463-498 